\title{
VANTAGENS DA CONSERVAÇÃO DA VEIA JUGULAR INTERNA NOS ESVAZIAMENTOS CERVICAIS UNILATERAIS
}

\section{ADVANTAGES OF INTERNAL JUGULAR VEIN PRESERVATION IN UNILATERAL NECK DISSECTIONS}

\author{
Marcelo Benedito Menezes ${ }^{1}$ \\ Abrão Rapoport, TCBC ${ }^{1}$ \\ Antônio Sérgio Fava ${ }^{1}$ \\ Carlos Neutzling Lehn
}

\begin{abstract}
RESUMO: Objetivo: Diante da dúvida sobre a existência ou não de vantagens relacionadas à conservação da veia jugular interna nos esvaziamentos cervicais unilaterais resolvemos observar se os pacientes que a tem sacrificada apresentam alterações ao exame de fundo de olho que indiquem um aumento da pressão intracraniana, avaliar a presença de alterações neurológicas e verificar as vantagens na evolução clínico-cirúrgica dos pacientes que tiveram a sua veia jugular interna conservada. Método: Trata-se de estudo prospectivo, não randomizado, de 15 pacientes portadores de carcinoma epidermóide da cabeça e pescoço, atendidos e operados no Serviço de Cirurgia de Cabeça e Pescoço do Hospital Heliópolis, que tiveram como parte de seu tratamento a realização de esvaziamento cervical completo (funcional ou não) unilateral, divididos em um grupo com ressecção da veia jugular interna e outro com sua conservação. Todos foram submetidos a exames de retinografia pré e pós-operatórios, além de avaliação neurológica e clínica. Resultados: Não ocorreram alterações oftalmológicas detectáveis através da retinografia em nenhum dos grupos. O grupo que teve sua veia jugular interna ressecada apresentou maior incidência de queixas neurológicas no pós-operatório, além de uma evolução cirúrgica mais desfavorável, com um maior período de internação devido às complicações locais apresentadas. Conclusões: A conservação da veia jugular interna nos esvaziamentos cervicais unilaterais beneficia a evolução pós-operatória dos pacientes, com um menor período de internação, apesar de não termos identificado qualquer evidência pós-operatória de aumento da pressão intracraniana nos pacientes que tiveram a veia ligada.
\end{abstract}

Descritores: Esvaziamento cervical radical — efeitos adversos; Veias jugulares; Hipertensão intracraniana benigna.

\section{INTRODUÇÃO}

O carcinoma espinocelular de cabeça e pescoço dissemina-se com freqüência para linfonodos cervicais. $\mathrm{O}$ tratamento de eleição é o cirúrgico, através do esvaziamento cervical, que muitas vezes é executado em monobloco, ou seja, em peça única, juntamente com a ressecção da lesão primária.

Desde que a técnica para a realização do esvaziamento cervical foi padronizada ${ }^{1}$, esta sofreu algumas mudanças significativas, sempre na tentativa de, mantendo-se o rigor oncológico, diminuir os índices de morbidade e mortalidade decorrentes desse procedimento, chegando à conservação de todas as estruturas não linfáticas: o músculo esternocleidomastóideo, a veia jugular interna e o nervo espinal acessório. Para isto, baseou-se em estudos anatômicos que demonstraram não existir relação entre os vasos linfáticos cervicais e estas estruturas ${ }^{2}$. Quando comparados aos resultados históricos obtidos com o esvaziamento descrito por Crile, os resultados com a técnica mais conservadora foram semelhantes, passando a ser difundido pelo mundo ${ }^{3}$.

As conseqüências da ligadura das veias jugulares internas foram analisadas em poucos estudos, nos quais foram observados quadros de hipertensão intracraniana com associação ou não de alterações oftalmológicas, es-

1. Cirurgião do Serviço de Cirurgia de Cabeça e Pescoço do Hospital Heliópolis, Hosphel, São Paulo, Brasil.

2. Chefe do Serviço de Cirurgia de Cabeça e Pescoço do Hospital Heliópolis, Hosphel, São Paulo, Brasil.

Recebido em 26/10/2001

Aceito para publicação em 18/06/2002

Trabalho realizado no Serviço de Cirurgia de Cabeça e Pescoço do Hospital Heliópolis e Curso de Pós-graduação em Cirurgia de Cabeça e Pescoço do Hospital Heliópolis, Hosphel, São Paulo. (Pesquisa com auxílio CAPES.) 
pecialmente nos esvaziamentos cervicais bilaterais com sacrifício de ambas as veias jugulares internas ${ }^{4}$. Outros autores relataram casos de ligadura unilateral da veia em que foi comprovada a instalação de hipertensão intracraniana no pós-operatório e alguns conseguiram demonstrar alterações anatômicas, tais como a atresia do seio lateral ou grandes diferenças entre os calibres dos forames jugulares, que predispunham determinados pacientes a desenvolverem este quadro ${ }^{5,6}$.

Apesar de estar relativamente claro que a conservação de uma das veias jugulares nos esvaziamentos bilaterais é benéfica, evitando a hipertensão intracraniana severa e suas consequiências, pouco se sabe sobre os benefícios da conservação da veia jugular interna nos esvaziamentos cervicais unilaterais. Benefícios potenciais podem incluir a diminuição do edema facial e, principalmente, cerebral, alterando a morbidade pós-operatória ${ }^{7-9}$.

Este estudo se propõe a avaliar a existência de alterações no exame de fundo de olho nos pacientes que tiveram a veia jugular interna sacrificada durante o esvaziamento cervical unilateral e a verificar a presença de alterações neurológicas que possam estar relacionadas à ligadura, além de comparar a evolução clínico-cirúrgica entre os pacientes que tiveram a veia jugular interna ligada e aqueles que não.

\section{MÉTODO}

No período de fevereiro de 1996 a janeiro de 1997, 15 pacientes incluídos neste estudo foram atendidos no Serviço de Cirurgia de Cabeça e Pescoço do Hospital Heliópolis, São Paulo, Brasil. Todos do sexo masculino, com idade variando de 37 a 69 anos, com média de 53,4 anos e mediana de 54 anos. Destes, 12 pacientes eram brancos e três negros.

Todos os pacientes eram portadores de carcinoma epidermóide da região da cabeça e pescoço. Seis deles apresentavam tumor primário na laringe, três pacientes com primário de hipofaringe, três com primário na boca e quatro com primário localizado na orofaringe. Apenas um deles era estádio T1, seis eram T2, cinco T3 e três eram T4. Quanto ao estádio N, cinco eram N0, cinco N1, três $\mathrm{N} 2$ e apenas dois N3.

Todos os pacientes foram previamente esclarecidos quanto à finalidade do estudo e informados que, exceto pelas retinografias, não haveria qualquer alteração na terapêutica proposta inicialmente. Todos assinaram termo de aceitação para a inclusão no trabalho, assim como obtivemos a concordância de, ao menos, um familiar.

Trata-se de estudo prospectivo e não randomizado de pacientes portadores de carcinoma espinocelular de cabeça e pescoço, com indicação de esvaziamento cervical unilateral em "monobloco" com a ressecção da lesão primária. Os pacientes foram consecutivos a partir da data de início do estudo e a opção pela ligadura ou não da veia jugular interna se deu no intra-operatório, levando-se em conta, principalmente, o fator oncológico e técnico para esta decisão. Desta forma, somente foram conservadas as veias onde não havia dúvida quanto à ausência de contato da mesma com linfonodos metastáticos e também que os linfonodos não apresentassem ruptura capsular macroscópica. Foram excluídos os casos em que durante a cirurgia, constatou-se a trombose prévia da veia jugular, fosse através de compressão ou invasão da mesma pela metástase, fosse por qualquer outro motivo.

Nos períodos pré e pós-operatório, os pacientes foram submetidos a avaliações clínicas diárias, iniciando avaliação do estado geral através da escala de Karnofsky e atualização do estadiamento clínico. Após a cirurgia foram reavaliados os parâmetros analisados e identificada a presença de alterações locais relacionadas à cirurgia (edema, infecção, dor, integridade das suturas).

Todos os pacientes foram interrogados sobre sintomas não relacionados à neoplasia, com especial ênfase a possíveis distúrbios neurológicos preexistentes. História pregressa de cefaléia e/ou vertigens foram devidamente anotadas. Todos foram submetidos a exames neurológicos um a três dias antes da cirurgia, a fim de identificar possíveis alterações motoras ou sensitivas preexistentes. Esta avaliação foi repetida sistematicamente após a cirurgia, diariamente nos primeiros cinco dias, sendo continuado naqueles casos em que encontramos alguma alteração. Especial atenção foi dada às manifestações que pudessem estar relacionadas ao aumento da pressão intracraniana.

Todos os pacientes submeteram-se a exames oftalmológicos pré e pós-operatórios, constando de tonometria ocular (medição indireta da pressão dos líquidos intra-oculares) e retinografia, observando-se que o exame após a cirurgia fosse realizado num período de, no máximo, cinco dias após o ato operatório. As retinografias foram obtidas após uso de colírio cicloplégico para dilatação das pupilas, melhorando as condições para o exame. Foram obtidas pelo menos quatro fotografias de cada fundo de olho, focalizando especialmente a área da papila oftálmica. Todos os exames foram realizados no consultório do Grupo de Retina da Cadeira de Oftalmologia da Faculdade de Medicina da Fundação ABC.

As alterações encontradas em cada um dos exames foram devidamente anotadas, inclusive aquelas encontradas nos exames pré-operatórios, possibilitando o estudo comparativo entre eles e evitando a identificação de falsas alterações no pós-cirúrgico. No exame pós-operatório, procuramos principalmente por alterações como edema de papila ou outros sinais indiretos de surgimento deste edema, tais como aumento da angulação dos vasos retinianos em sua emergência na papila ou o ingurgitamento das veias retinianas.

Todas as imagens obtidas foram, posteriormente, comparadas uma a uma, inclusive utilizando técnicas de estereofotografia, a fim de identificar mínimas alterações existentes entre as imagens do pré e do pós-operatório em cada olho, de cada paciente. A técnica de estereofotografia consiste na obtenção de imagens tridimensionais das papilas oftálmicas através da superposição binocular de duas imagens semelhantes obtidas de uma mesma papila, num mesmo momento.

A análise estatística foi feita utilizando o teste exato de Fisher sendo os valores de "p" considerados signifi- 
cantes quando $\mathrm{p}<0,05$. As variáveis utilizadas foram a presença de edema após a cirurgia, a ocorrência de complicações locais e de neurológicas.

\section{RESULTADOS}

Os pacientes foram distribuídos em GRUPO A (formado pelos pacientes que tiveram sua jugular interna ligada), com um total de nove pacientes e em GRUPO B (pacientes que tiveram a veia conservada) com seis pacientes. A média de idade do grupo A foi de 54,4 anos e do grupo B de 51,8 anos. Quanto ao estádio "T", a maioria dos pacientes encontrava-se no estádio 2 e $3(77,8 \%)$ enquanto apenas um era T1 e outro T4. Enquanto isto, no grupo $\mathrm{B}, 83,3 \%$ eram T3 ou T4 e o restante, T2. Quanto ao "N", no grupo A predominaram pacientes $\mathrm{N} 2$ e N3, enquanto no B, casos N0 e N1.

No pós-operatório imediato um paciente faleceu, e o mesmo havia sido submetido à ligadura da veia jugular interna. Este paciente não foi submetido à autópsia, não tendo sido possível relacionar diretamente sua evolução à ligadura ou não da veia jugular. Dentro do grupo dos pacientes submetidos à ligadura da veia jugular interna, este paciente será analisado como sendo parte do mesmo e como tendo tido uma evolução desfavorável.

A Tabela 1 mostra a incidência dos sintomas neurológicos avaliados em cada um dos grupos, com uma maior incidência destes sintomas no grupo com sacrifício da veia jugular interna (GRUPO A). Neste grupo, cinco pacientes $(62,5 \%)$ apresentaram queixas neurológicas, enquanto no GRUPO B, dois pacientes $(33,3 \%)$ apresentaram este mesmo tipo de queixas.

A Tabela 2 mostra a incidência de edema facial em cada grupo de pacientes, considerando desde a ausência deste, até a presença de edema intenso, acometendo inclusive a região peri-orbitária.

A Tabela 3 traz as complicações locais pós-operatórias ocorridas em cada grupo, ainda no período em que o paciente permaneceu internado.

Tabela 1

Manifestações neurológicas apresentadas pelos pacientes dos dois grupos nos primeiros dias após a cirurgia

\begin{tabular}{l|c|c}
\hline Sintomas neurológicos & $\begin{array}{c}\text { Grupo A } \\
(n=9)\end{array}$ & $\begin{array}{c}\text { Grupo B } \\
(n=6)\end{array}$ \\
\hline $\begin{array}{l}\text { Vertigem } \\
\text { Cefaléia }\end{array}$ & $3(33,3 \%)$ & 0 \\
Vertigem e cefaléia & 0 & $1(16,7 \%)$ \\
Cefaléia e alteração & $1(11,1 \%)$ & $1 \quad(16,7 \%)$ \\
no comportamento & $1(11,1 \%)$ & 0 \\
\hline Total & $5(55,6 \%)$ & $2(33,3 \%)$ \\
\hline
\end{tabular}

* Um paciente do GRUPO A faleceu no pós-operatório imediato, o que não pode ser diretamente relacionado a aumento da pressão intracraniana.
Tabela 2

Edema pós-operatório em cada grupo

\begin{tabular}{l|c|c}
\hline Edema & Grupo A (\%) & \multicolumn{2}{|c}{ Grupo B } \\
\hline I-/ & 0 & $3(50,0 \%)$ \\
+ & $4(50,0 \%)$ & $1(16,7 \%)$ \\
++ & $3(37,5 \%)$ & $2(33,3 \%)$ \\
+++ & $1(12,5 \%)$ & 0 \\
\hline Total & $8(100 \%) *$ & $6(100 \%)$ \\
\hline
\end{tabular}

*Obs.: o paciente que faleceu no pós-operatório imediato foi excluído.

$* * /-/=$ s/ edema $;+=$ edema discreto $;++=$ edema moderado; $+++=$ edema intenso

Tabela 3

Complicações locais pós-operatórias

\begin{tabular}{|c|c|c|c|c|}
\hline & Grupo A & Grupo B & & Total \\
\hline Sem complicações & $5 \quad(55,5 \%)$ & $5 \quad(83,3 \%)$ & 10 & $(66,7 \%)$ \\
\hline Fístula & $2(22,2 \%)$ & 0 & 2 & $(13,3 \%)$ \\
\hline Deiscência de sutura & $1(11,1 \%)$ & 0 & 1 & $(6,7 \%)$ \\
\hline $\begin{array}{l}\text { Isquemia das bordas } \\
\text { da ferida }\end{array}$ & 0 & $1 \quad(16,7 \%)$ & 1 & $(6,7 \%)$ \\
\hline Morte & $1 \quad(11,1 \%)$ & 0 & 1 & $(6,7 \%)$ \\
\hline Total & $9(100 \%)$ & $6(100 \%)$ & 15 & $(100 \%)$ \\
\hline
\end{tabular}

O Gráfico 1 representa cada um dos pacientes de cada grupo e o período que permaneceram internados após a cirurgia.

A Tabela 4 mostra a relação existente entre a ligadura da veia jugular interna e a presença de alterações neurológicas e de complicações locais, com maior incidência destas eventualidades nos pacientes submetidos à ligadura da veia jugular interna. Mostra ainda um maior período de hospitalização entre estes pacientes.

As retinografias obtidas no pré e pós-operatório de cada paciente não evidenciaram qualquer alteração. Algumas alterações foram detectadas na retina de alguns pacientes, mas todas presentes já no pré-operatório, ou seja, estes pacientes apresentavam alterações no exame de fundo de olho que, caso não tivessem sido registradas anteriormente à cirurgia, poderiam ser confundidas com edema de papila (mielinização das fibras do nervo óptico, como apresentado por um dos pacientes e que se trata de um pseudo-edema).

$\mathrm{Na}$ análise estatística, nenhum dos parâmetros estudados (complicações pós-operatórias, edema, manifestações neurológicas e período de internação após a cirurgia) apresentou diferença estatisticamente significante. 


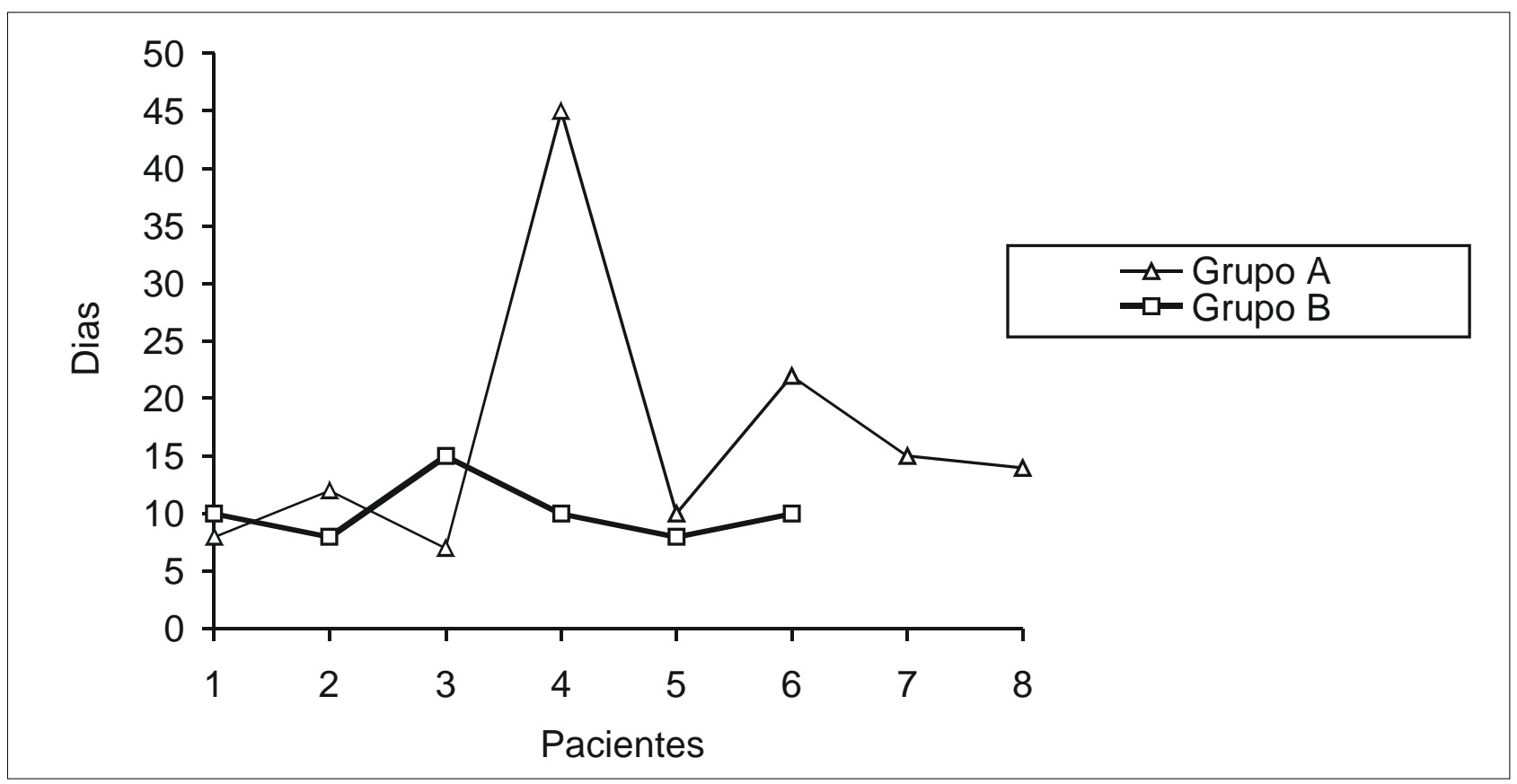

Gráfico 1 - Representa os dias de internação de cada paciente em cada grupo.

Tabela 4

Incidência de complicações pós-operatórias, alterações neurológicas e médias de internação em cada grupo

\begin{tabular}{l|c|c}
\hline Evolução & $\begin{array}{c}\text { Grupo A } \\
(n=9)\end{array}$ & $\begin{array}{c}\text { Grupo B } \\
(n=6)\end{array}$ \\
\hline Alterações neurológicas & $6(66,7 \%)$ & $2(33,3 \%)$ \\
Complicações locais & $3(33,3 \%)$ & $1(16,7 \%)$ \\
Média de hospitalização & 16,7 days & 10,1
\end{tabular}

\section{DISCUSSÃO}

O esvaziamento cervical freqüentemente faz parte da indicação cirúrgica para o tratamento do câncer de cabeça e pescoço. Raramente se dá grande importância ao fato de conservarmos ou não a veia jugular interna durante sua execução. Há a idéia de que o sacrifício unilateral desta estrutura não traz prejuízo significativo ao paciente, já que o retorno venoso pode ser feito por vias alternativas, principalmente através do plexo prévertebral, que seria mais importante, inclusive, que a veia jugular interna oposta ${ }^{10}$.

Quando recorremos à literatura sobre o assunto, encontramos relatos esporádicos que identificam alguma manifestação danosa ao paciente relacionada ao sacrifício da veia jugular interna e não encontramos nenhum estudo que demonstrasse que tal procedimento é realmente irrelevante na evolução do paciente.

Quando Crile (1906) descreveu a técnica do esvaziamento cervical, indicando-a para o tratamento da extensão linfonodal dos carcinomas de cabeça e pescoço, a única preocupação demonstrada foi com os fatores oncológicos, tentando atingir a extirpação radical do câncer. A proposta parecia o máximo que a cirurgia poderia fazer por estes pacientes, apesar das conseqüentes seqüelas envolvidas. A intensidade das seqüelas e a emergência da radioterapia como uma alternativa menos mutilante e arriscada (devemos lembrar que a anestesia e a antibioticoterapia eram rudimentares até a metade do século XX deixaram o esvaziamento cervical como um último recurso para o tratamento. Assim, durante o longo período compreendido entre o trabalho de Crile (1906) e o ano de 1951, pouco se discutiu sobre a técnica operatória do esvaziamento cervical e sua importância. A principal discussão envolvia a indicação do tratamento cirúrgico ou radioterápico para o tratamento das neoplasias de cabeça e pescoço.

Já não se discutia a possibilidade de um procedimento cirúrgico mais radical, mas sim um meio de minimizar as seqüelas ou justificá-las, admitindo-se não haver alternativa terapêutica mais satisfatória quanto ao controle da neoplasia.

Vários autores passaram a valorizar a incapacitação gerada pela ressecção do nervo espinal ${ }^{11}$ e começaram a surgir idéias de que em alguns casos a conservação deste nervo não seria importante para a sobrevida, assim como a preservação de outras estruturas não linfáticas.

Apesar das seqüelas deixadas pelo esvaziamento cervical terem sido bastante estudadas, geralmente o foram quanto à reabilitação tardia do paciente e a sua reintegração às atividades que desenvolvia antes da doença. Raramente houve preocupação com as diferenças precoces entre as cirurgias mais agressivas e os procedimentos mais 
conservadores, fosse quanto a sinais e sintomas apresentados durante os primeiros dias após a cirurgia, a presença de complicações ou, ainda, quanto ao tempo de internação após a cirurgia.

A partir da década de 50, alterações na pressão intracraniana após a ligadura de uma das veias jugulares internas foram descritas em modelos experimentais ${ }^{6,12}$ enquanto alguns autores suspeitavam desta alteração diante da evolução de seus pacientes após cirurgias em que apenas uma das veias jugulares foi ressecada ${ }^{13-15}$, que apresentavam desde cefaléia até manifestações neurológicas mais significativas, como vertigens e alterações de comportamento, até óbitos que estariam relacionados à hipertensão intracraniana ${ }^{14}$. Outra manifestação descrita foi a alteração visual, relacionada ao surgimento de edema da papila oftálmica ${ }^{8,13,16}$.

$\mathrm{Na}$ tentativa de demonstrar a relação entre a ligadura de uma das veias jugulares e o aumento da pressão intracraniana, chegou-se a realizar monitoração contínua deste parâmetro, através de punção lombar, em pacientes submetidos a esvaziamento cervical unilateral ${ }^{7,14,15}$.

Contrapondo-se aos relatos anteriores, em nosso estudo, apesar de, teoricamente, dispormos de condições técnicas mais favoráveis para a realização de exames do fundo de olho, nossos resultados não mostraram qualquer alteração retiniana, especialmente quanto à presença de edema de papila, em exames realizados até o quinto dia de pós-operatório. Não somente fizemos uma análise momentânea do aspecto da retina, sempre utilizando a dilatação farmacológica das pupilas e de equipamento com capacidade de ampliação, mas também realizamos fotografias das retinas no pré e pós-operatório, o que nos possibilitou uma análise cuidadosa e comparativa entre as imagens obtidas, mesmo vários dias após os exames terem sido realizados, além de podermos solicitar diferentes opiniões e realizar comparações utilizando a técnica de estereofotografia. Apesar disto, não encontramos qualquer alteração oftalmológica sugestiva de aumento da pressão intracraniana, fosse um papiledema evidente ou o maior ingurgitamento venoso retiniano que pudesse sugerir um edema ou pelo menos um aumento da pressão venosa intra-ocular.

Talvez a explicação para que não encontrássemos alterações neste grupo de pacientes esteja em estudo no qual a causa da hipertensão intracraniana foi identificada como sendo a ausência congênita do seio transverso oposto ao lado da ligadura ${ }^{17}$. Esta eventualidade tornaria a dificuldade para o retorno venoso similar ao observado diante da ligadura bilateral da veia jugular interna, já que esta pode ser considerada como uma extensão anatômica do seio transverso ipsilateral ${ }^{14,18}$.
No que se refere às alterações clínicas relacionadas com a ligadura da veia jugular interna, tivemos diferenças, quanto às complicações, entre os dois grupos. Entre os pacientes que foram submetidos à ligadura da veia jugular interna, nota-se um maior número de complicações do procedimento cirúrgico como um todo $(33,3 \%$ contra $16,7 \%$ no grupo sem ligadura). Estas complicações fizeram com que os pacientes deste grupo ficassem internados por um período maior (média de 16,7 dias contra 10,1 dias do outro grupo). Não vimos em nenhum outro estudo uma comparação do índice de complicações pós-operatórias que levasse em conta todo o procedimento cirúrgico e não só o esvaziamento cervical.

Importante notar que a maioria dos pacientes apresentou algum grau de edema facial após a cirurgia: todos do grupo com ligadura da veia e três $(50 \%)$ entre os que a tiveram conservada. Obviamente, diante do pequeno número de casos que estamos apresentando, não podemos concluir que esta diferença foi diretamente causada pela ligadura ou não da veia jugular interna, porém, trata-se de um forte indício desta eventualidade.

Quanto às alterações neurológicas observadas em nossos pacientes, elas se restringiram a quadros de vertigens e cefaléia, em alguns casos concomitantes, em outros separadas. No grupo submetido à ligadura da veia jugular interna, 62,5\% dos pacientes apresentaram queixas neurológicas enquanto no grupo que a teve conservada, apenas 33,3\% dos pacientes evoluíram com este tipo de queixa. Não é possível, com estes dados, estabelecer uma ligação direta entre a ligadura da veia e a presença destas alterações ou, menos ainda, relacioná-las à hipertensão intracraniana, afinal não encontramos sinais objetivos desta entidade em nenhum de nossos pacientes. Podemos dizer apenas que há indícios de vantagens na evolução pós-operatória dos pacientes que têm o retorno venoso do segmento cefálico mantido através das veias jugulares internas. Estas manifestações neurológicas vão ao encontro das descritas por diversos autores que também se preocuparam com este problema ${ }^{5,7,14}$.

Quanto às complicações após a cirurgia, em nosso estudo encontramos um maior índice de complicações e, conseqüentemente, um maior período de internação no grupo de pacientes submetidos à ligadura de uma das veias jugulares internas, o que acaba indo contra ao observado por outros autores ${ }^{19}$.

Concluímos que a conservação da veia jugular interna nos esvaziamentos cervicais unilaterais parece beneficiar a boa evolução dos pacientes, com um menor período de internação, apesar de não termos encontrado evidências do aumento de pressão intracraniana através do estudo retinográfico. 


\begin{abstract}
Background: Facing the doubt about the existence or not of advantages in preserving the internal jugular vein (IJV) in unilateral neck dissections, the authors decided to determine if the patients with IJV ligation develop changes in retina examination that indicate an increasing in intracranial pressure, to evaluate the presence of neurologic changes and to verify the advantages on clinical and surgical outcome of the patients with IJV ligation. Method: This is a non-radomized, prospective study comprising 15 patients with head and neck squamous carcinoma treated at the Head and Neck Service of Hospital Heliópolis who were submitted to unilateral neck dissection as part of their treatment and divided in two groups: one with ligation of IJV and other with its preservation. All underwent to preoperative and postoperative retinography and clinical and neurological evaluation. Results: Detectable ophthalmologic changes occurred in none of the two groups. The group with IJV ligation presented a larger incidence of neurologic complaints in postoperative period and a less favorable surgical outcome with a larger period of hospitalization due by local complications. Conclusions: Preservation of IJV in unilateral neck dissections took to a better postoperative outcome with less time of hospitalization even though no evidence of postoperative increasing in intracranial pressure was observed in patients with IJV ligation.
\end{abstract}

Key Words: Radical neck dissection - adverse effects; Jugular veins; Benign intracranial hypertension.

\section{REFERÊNCIAS}

1. Crile G. Excision of Cancer of the Head and Neck. JAMA, 1906; 47: 1780-6.

2. Suarez O. El Problema de las Metastasis Linfaticas y Alejadas del Cancer de Laringe e Hipofaringe. Rev Otorrinolaringol, 1963; 23:83-99.

3. Bocca E, Pignataro O. A Conservation Technique in Radical Neck Dissection. Ann Otol Laryngol, 1967; 76: 975-87.

4. Rucker CW. Defects in The Visual Fields Resulting From Increased Intracranial Pressure. New York J Med, 1949; 49:2417-21.

5. Fitz-Hugh GS, Robins RB, Craddock WD. Increased Intracranial Pressure Complicating Unilateral Neck Dissection. Laryngoscope, 1966; 76: 893-906.

6. Kawajiri H, Furuse M, Namba R, Kotani J, Oka T. Effect of Internal Jugular Vein Ligation on Resorption of Cerebrospinal Fluid. J Max-Fac Surg, 1983; 11:42-5.

7. Royster HP. The Relation Between Internal Jugular Vein Pressure and Cerebrospinal Fluid Pressure in The Operation of Radical Neck Dissection. Ann Surg, 1953; 135: 826-32.

8. Morfit HM, Cleveland H. Permanent Increased Intracranial Pressure Following Unilateral Radical Neck Dissection. Arch Surg, 1958; 76: 713-23.

9. Tobin HA. Increased Cerebrospinal Fluid Pressure Following Unilateral Radical Neck Dissection. Laryngoscope, 1972; 82: 817-20.

10. Batson DV. Anatomic Problems Concerned in the Study of Blood Flow. Federation Proc, 1944; 4: 139-44.

11. Nahum AM, Mullally W, Marmor L. A Syndrome Resulting from Radical Neck Dissection. Arch Otolaryngol, 1961; 74: 424-8.

12. Kotani J, Nitta K, Sakuma Y, Sugioka S, Fugita N, Ueda Y. Effects of Bilateral Jugular Vein Ligation on Intracranial Pressure and Cerebrospinal Fluid Outflow Resistance in Cats. Br J Oral Max-Fac Surg, 1992; $30: 171-3$.
13. Swift GW. The Transverse Sinus and its Relation to Choked Disk. Arch Ophthalmol, 1930; 3: 47-70.

14. Jones RK. Increased Intracranial Pressure Following Radical Neck Surgery. Arch Surg, 1951; 63:599-603.

15. Sugarbaker ED, Wiley HM. Intracranial Pressure Studies Incident to Resection of the Internal Jugular Veins. Cancer, 1951; 4:242-50.

16. De Vries WAEJ, Balm AJM, Tiwari RM. Intracranial Hypertension Following Neck Dissection. J Laryngol Otol, 1986; 100: 1427-31.

17. Lydiatt DD, Ogren F, Lydiatt WM, Hahn FJ. Increased Intracranial Pressure as Complication of Unilateral Radical Neck Dissection in a Patient with Congenital Absence of the Transverse Sinus. Head Neck Surg, 1991; 13: 359-62.

18. Waltner JG. Anatomic Variations of the Lateral and Sigmoid Sinuses. Arch Otolaryngol, 1944; 39: 307-12.

19. Molinari R, Cantù G, Chiesa F, Grandi C. Retrospective Comparision of Conservative and Radical Neck Dissection in Laryngeal Cancer. Ann Otol Rhinol Laryngol, 1980; 89: 578-581.

Endereço para correspondência:

Prof. Dr. Abrão Rapoport

Pça. Amadeu Amaral, 47 - cj. 82 - Paraíso

01327-010 — São Paulo-SP

E-mail: cpgcp.hosphel@attglobal.net 